\title{
13. Exacerbaciones agudas en pacientes con fibrosis pulmonar idiopática
}

\author{
ARTURO MORALES S.* y JUAN CARLOS RODRÍGUEZ D.**
}

\section{Acute exacerbations in patients with idiopathic pulmonary fibrosis}

Classically we understand as an exacerbation of Idiopathic Pulmonary Fibrosis (IPF) to a clinically significant respiratory deterioration, without obvious cause. At present, the concept of "acute triggered exacerbations" is preferred to refer to those that are generated in the context of infection, aspiration, drug toxicity, pulmonary thromboembolism, heart failure or after invasive procedures. While the term "idiopathic acute exacerbations" is reserved for those in which we do not find a trigger. The prognosis is ominous and the mortality is high, with figures that fluctuate between 50 to $90 \%$ depending on the need for ventilatory support. Many times an acute exacerbation can be the final event of a patient with IPF. The treatment is not entirely clear, there is no robust evidence of the benefit of therapies, historically corticosteroids have been used as standard therapy, however current evidence questions the benefits of such a treatment.

Key words: Idiopathic pulmonary fibrosis; Pulmonary Embolism; Heart Failure.

\section{Resumen}

Clásicamente entendemos como exacerbación de la Fibrosis pulmonar idiopática (FPI) a un deterioro respiratorio, clínicamente significativo, sin causa evidente. En la actualidad se prefiere el concepto de "exacerbación aguda gatillada" para referirnos a aquella que se genera en el contexto de infección, aspiración, toxicidad por drogas, tromboembolismo pulmonar, insuficiencia cardiaca o posterior a procedimientos invasivos. Mientras que se reserva el termino de "exacerbación aguda idiopática" a aquella en la que no encontramos un gatillante. El pronóstico es ominoso, con mortalidad elevada, con cifras que fluctúan entre 50-90\% dependiendo de la necesidad de soporte ventilatorio. Por lo que muchas veces una exacerbación aguda puede ser el evento final de un paciente con FPI. El tratamiento no es del todo claro, no existe evidencia robusta del beneficio de terapias, históricamente los corticoides se han utilizados como terapia estándar, sin embargo la evidencia actual cuestiona los beneficios de dicho tratamiento.

Palabras clave: Fibrosis pulmonar idiopática; Embolía pulmonar; Insuficiencia Cardíaca.

Antes de plantear el manejo de las exacerbaciones en pacientes con fibrosis pulmonar idiopática (FPI), creemos que es importante unificar conceptos con respecto a cómo se definen dichos eventos, cuál es la incidencia de ellos y a las presuntas etiologías y factores de riesgo asociados. Ello nos permitirá comprender mejor la aproximación global que debemos tener al enfrentarnos a estos pacientes.

\section{Definición}

Clásicamente entendemos la exacerbación de una FPI cuando existe un deterioro respiratorio, clínicamente significativo, sin causa evidente, que reúna los siguientes criterios:

a) Aumento significativo de la disnea de menos de un mes de evolución.

* Clínica Alemana de Santiago e Instituto Nacional del Cáncer. Santiago, Chile.

** Instituto Nacional del Tórax y Clínica Las Condes. Santiago, Chile. 
b) Presencia de nuevas alteraciones en TC de tórax (ej, opacidades en vidrio esmerilado bilateral o consolidaciones) sobre un trasfondo de patrón de neumonia intesrticial usual: NIU.

c) Exclusión de diagnósticos alternativos (ej. infección, falla cardíaca, o tromboembolismo pulmonar).

Estos criterios fueron establecidos en el año 2007, y la gran mayoría de los estudios sobre tratamiento que expondremos más delante de basaron en ellos para definir una exacerbación ${ }^{1}$. En los años 2015 y 2016 se propuso modificar estos criterios, flexibilizando el tiempo de duración del deterioro de la disnea y eliminando la exigencia de exclusión de infección. Es así como actualmente se denomina "exacerbaciones agudas gatilladas" a aquellas que se generan en el contexto de infección, aspiración, posterior a procedimientos y cirugías, y a toxicidad por drogas. Mientras que se reserva el termino de "exacerbaciones agudas idiopáticas" a aquellas en las que no encontramos un gatillante ${ }^{2}$.

\section{Incidencia}

La incidencia anual varía entre 1 a 20\% según la serie reportada. En FPI en estadios iniciales la incidencia es más baja. Datos provenientes de un meta-análisis reporta una incidencia de 4,1 exacerbaciones por cada 100 pacientes-año. Otros estudios informan de incidencias de hasta 20 eventos por 100 pacientes-año. Las exacerbaciones estadísticamente son más comunes durante los meses de invierno y son más frecuentes en pacientes con enfermedad avanzada y bajo tratamiento inmunosupresor.

\section{Etiología}

Es aún desconocida. Se postula que podría ser la respuesta a un evento externo (ej infección, microaspiración) que genere injuria pulmonar aguda.

\section{Factores de riesgo}

- FPI en etapas avanzadas.

- Caída reciente de CVF ( $\geq 10 \%$ en 6 meses).

- Historia de exacerbaciones previas.

- Índice de masa corporal alto.

\section{Manejo}

No existe una terapia efectiva demostrada para el manejo de las exacerbaciones de FPI, por lo que hasta ahora el manejo se basa en intervenciones que carecen de respaldo científico robusto. Dentro de las opciones terapéuticas de las exacerbaciones de FPI uno de los fármacos que siempre se considera emplear es el corticoide; aún cuando no existe evidencia científica sólida tras ésta recomendación. Esta terapia está respaldada sólo por trabajos de cohorte no controlados y por reportes anecdóticos de beneficio, por lo que las actuales guías clínicas asignan una recomendación débil para este tratamiento. Al tomar en consideración las altas tasas de mortalidad asociadas a una exacerbación de FPI se entiende el amplio empleo de estos fármacos. Se debe tener en consideración eso sí que no es posible determinar si la mejoría clínica al emplear corticoides se debe al fármaco en sí, o a la historia natural de la enfermedad sin poder descartar otros factores no medidos. El terreno es aún más sombrío cuando intentamos establecer pautas con respecto a qué dosis de corticoide emplear, y a si se deben o no utilizar éstos en pulsos. Toda esta incertidumbre se debe a la carencia de estudios randomizados que respalden estas conductas.

Estudios observacionales han evaluado el uso de rituximab con plasmaféresis e inmunoglobulinas, ciclosporina, tacrólimus, y trombomodulina, sumado a los corticoides. Hasta ahora la gran mayoría de estos estudios tiene reparos metodológicos que impiden obtener conclusiones sólidas a partir de ellos, por ende cualquier empleo de dichos fármacos hoy en día debe ser considerado como una estrategia de rescate sin respaldo en la evidencia científica actual.

Aún cuando la mayoría de estos pacientes recibe antibióticos sistémicos, no existe una evidencia científica clara que apoye esta estrategia. El empleo de los niveles séricos de procalcitonina como ayuda en discernir sobre el uso de tratamiento antibiótico durante una exacerbación, ha reducido la indicación y duración de los cursos antibióticos. Sin embargo, los estudios que respaldan estas afirmaciones cuentan con sesgos metodológicos en sus diseños. El lavado broncoalveolar es frecuentemente utilizado en estos pacientes para descartar infección cuando las estrategias diagnósticas no invasivas resultan negativas, sin embargo, éste muchas veces la situación clínica del paciente no lo permite y otras veces puede ser de riesgo en pacientes lábiles en quienes podrían tolerar mal una broncoscopía por insuficiencia respiratoria catastrófica, por lo que no es poco frecuente el empleo de tratamiento antibiótico empírico.

El manejo de soporte debe incluir la paliación 
de síntomas, y corrección de la hipoxemia. Con respecto al uso de ventilación mecánica esta debe ser discutida caso a caso con los familiares, tomando en consideración que algunas series reportan mortalidad intrahospitalaria de hasta un $90 \%$ para estos pacientes cuando requieren ventilación invasiva. Al analizar estos datos es importante considerar que esta evidencia viene de estrategias de ventilación no protectoras por lo que nuevas estrategias podrían eventualmente mejorar algo la sobrevida intrahospitalaria. Si se determina el empleo de la ventilación mecánica ésta debe ser con estrategias protectoras (volúmenes corriente y presiones plateau bajas).

La prevención de las exacerbaciones agudas de FPI es a nuestro juicio lo más importante, dado la alta mortalidad asociada a éstas y la pobre respuesta al tratamiento actual. La estrategia principal de prevención involucra el evitar los gatillantes que puedan producir una exacerbación. El riesgo de infección puede disminuir tras vacunación. Con respecto al empleo de terapia antiácida un estudio retrospectivo sugirió un beneficio en cuanto a una menor tasa de exacerbaciones, pero sin asociarse a una menor tasa de hospitalización ni mortalidad, por lo que es necesario reevaluar éstos resultados en estudios randomizados. Si tomamos en consideración que las microaspiraciones pudiesen estar implicadas en la etiología de algunas exacerbaciones, esta estrategia toma mayor peso. Los nuevos fármacos para el tratamiento de la FPI también han demostrado reducción en las tasas de exacerbaciones de la FPI. La evidencia proveniente de ensayos clínicos randomizados que evaluaban el empleo de Nintedanib en el tratamiento de pacientes con FPI, reportan que tras su uso existe una reducción en el número de exacerbaciones (eso sí, en análisis de datos combinados y como resultado secundario). Los principales trabajos randomizados con pirfenidona no midieron este resultado. Sin embargo, evidencia proveniente de trabajos con menos casos sí ha demostrado que su uso podría reducir las exacerbaciones agudas de FPI, incluso las ocasionadas en contexto de cirugías torácicas.

\section{Pronóstico}

La mortalidad intrahospitalaria de una exacerbación aguda alcanza cifras de un 50\%, y alcanza hasta un $90 \%$ en caso de requerir soporte ventilatorio invasivo. La sobrevida promedio de los pacientes que presentan una exacerbación aguda en FPI es de sólo 3 a 4 meses. Un $46 \%$ del total de las muertes en pacientes con IPF son precedidas por una exacerbación.

En una exacerbación de FPI las variables que se asocian a un peor pronóstico son compromiso inflamatorio difuso en tomografía computada de alta resolución (TACAR), peores relaciones entre presión arterial de oxígeno y fracción inspirada de oxígeno, y valores basales bajos de las pruebas de función pulmonar ( $\mathrm{CVF}$ y $\left.\mathrm{DL}_{\mathrm{CO}}\right)$.

\section{Reflexión final}

Las exacerbaciones agudas de la FPI son una enfermedad de alta morbi-mortalidad, cuyo gatillante no está del todo aclarado. Las estrategias terapéuticas, por sobre las de soporte, son limitadas, y muchas consideran fármacos que no cuentan con una evidencia sólida de respaldo. Quizás lo más importante en el manejo de las exacerbaciones sea su prevención, evitando en lo posible los eventuales gatillantes.

\section{Bibliografía}

1.- COLLARD HR, MOORE BB, FLAHERTY KR, BROWN KK, KANER RJ, KING TE Jr, et al. Idiopathic Pulmonary Fibrosis Clinical Research Network Investigators. Acute exacerbations of idiopathic pulmonary fibrosis. Am J Respir Crit Care Med 2007; 176: 636-43.

2.- COLLARD HR, RYERSON CJ, CORTE TJ, JENKINS G, KONDOH Y, LEDERER DJ, et al. Acute Exacerbation of Idiopathic Pulmonary Fibrosis. An International Working Group Report. Am J Respir Crit Care Med 2016; 194: 265-75.

Correspondencia a:

Dr. Arturo Morales S.

Email: ajmorals@gmail.com 